\title{
Hepatocellular carcinoma cases with high levels of c-Raf-1 expression may benefit from postoperative adjuvant sorafenib after hepatic resection even with high risk of recurrence
}

\author{
Jianyong Lei ${ }^{1,2,5}$, Jinjing Zhong ${ }^{3}$, Jingcheng Hao ${ }^{2}$, Zhengni Liu ${ }^{2}$, Peng Zhang ${ }^{4}$, Lixue \\ $\mathrm{Wu}^{3}$, Lunan Yan ${ }^{2}$, Jinqiang Zhu ${ }^{1}$, Yong Zeng ${ }^{2}$, Bo $\mathrm{Li}^{2}$, Tianfu Wen ${ }^{2}$ and Wentao \\ Wang $^{2}$ \\ ${ }^{1}$ Thyroid and Parathyroid Surgery Center, West China Hospital of Sichuan University, Chengdu, China \\ ${ }^{2}$ Liver Surgery, West China Hospital of Sichuan University, Chengdu, China \\ ${ }^{3}$ Department of Pathology, West China Hospital of Sichuan University, Chengdu, China \\ ${ }^{4}$ Third General Surgery, The First Hospital of Handan, Handan, Hebei, China \\ ${ }^{5}$ State Key Laboratory of Biotherapy/Collaborative Innovation Center of Biotherapy, West China Hospital, Sichuan University, \\ Chengdu, China \\ Correspondence to: Wentao Wang, email: ljydoctor@163.com \\ Jinqiang Zhu, email: zjadoctor@163.com \\ Keywords: hepatocellular carcinoma, recurrence, sorafenib, risk factors, liver resection \\ Received: March 01, $2015 \quad$ Accepted: March 20, $2015 \quad$ Published: March 13, 2016 \\ This is an open-access article distributed under the terms of the Creative Commons Attribution License, which permits unrestricted use, \\ distribution, and reproduction in any medium, provided the original author and source are credited.
}

\section{ABSTRACT}

Background and Aims: Liver resection combined with postoperative sorafenib to prevent recurrence remains a controversial approach for cases of hepatocellular carcinoma (HCC), especially cases with a high risk of recurrence. This study aimed to investigate the efficacy and safety of liver resection combined with sorafenib for HCC with a high risk of recurrence.

Results: Most of the cases of HCC were caused by hepatitis B virus (HBV) infection ( 23 cases, 92\%). Most of these tumors ( 21 cases, $84 \%$ ) were stage III according to the TNM staging system ( 12 cases with IIIa, 9 cases with IIIb). In the months after hepatic resection, 19 of the 25 cases (76\%) were diagnosed with HCC recurrence or metastasis. Based on the tumor histological biomarker grading system, the group with higher expression levels of c-Raf-1 showed significantly longer overall survival than the group with lower expression of C-Raf-1 $(P=0.012)$. However, the long-term tumor-free survival advantage disappeared $(P=0.061)$. Univariate and multivariate analyses indicated that higher expression of c-Raf-1 was significantly associated with better overall survival (hazard ratio [HR]: 1.842; 95\% confidence interval [CI]: 1.211-2.542; $P=0.031$ ) and tumor-free survival (HR: $1.319 ; 95 \%$ CI: $1.017-1.543$; $P=0.046)$ in HCC patients who underwent radical hepatic resection.

Patients and Methods: We retrospectively collected 25 HCC cases with a high risk of recurrence who underwent radical liver resection and who took sorafenib postoperatively from Jan 2010 to Dec 2012. Factors that might contribute to tumor recurrence and treatment failure such as clinical factors, tumor features, and molecular biomarkers were included in our analysis.

Conclusions: HCC patients with a high risk of post-hepatic resection recurrence may benefit from postoperative sorafenib administered as an adjuvant therapy, especially in cases with high levels of c-Raf-1 expression on histological examination. 


\section{INTRODUCTION}

Hepatocellular carcinoma (HCC) is the sixth-most common type of cancer worldwide and the third-most common cause of cancer-related death [1]. Currently, local ablation, liver resection and liver transplantation offer the best chances for a cure. However, ablation can only be applied to small targets, and the liver graft shortage also limits the use of LT for HCC [2]. Thus, liver resection may be the best choice for early-stage HCC [3]. However, some studies have suggested that liver resection should also be applied to intermediate HCCs [4] and even in some advanced HCCs [5]. However, HCC recurrence and subsequent death are common [5, 6]. Many protocols have been used to prevent $\mathrm{HCC}$ recurrence, including postoperative adjuvant TACE $[7,8]$, hepatic arterial infusion therapy [9], administration of sorafenib [10], and other methods [11].

Sorafenib, a multikinase inhibitor, is thought to exert its antitumoral effects through the inhibition both angiogenesis and tumor cell proliferation by receptor tyrosine kinases, such as the serine-threonine kinases Raf1 , VEGFR1-3, and PDGFR- $\beta$. These agents have shown modest efficacy, resulting in improved survival inpatients with advanced HCC [12-14]. However, their utility in a postoperative adjuvant setting, especially for cases with a high risk of recurrence, is not known [12, 15]. Herein, we investigated the safety and efficacy of post-resection sorafenib for cases with a high risk of recurrence.

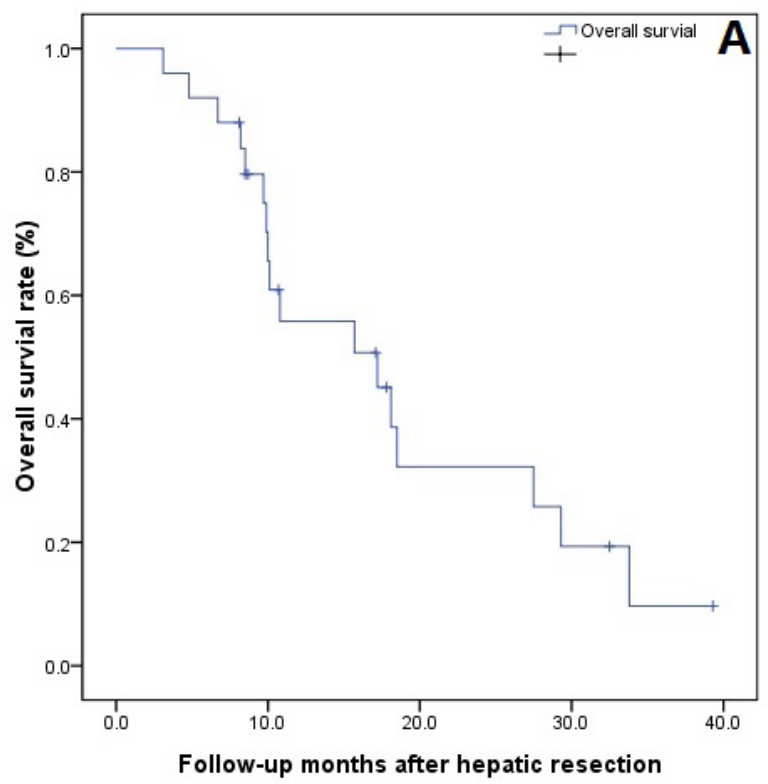

\section{RESULTS}

\section{Baseline characteristics}

As shown in Table 1, the baseline characteristics of the $25 \mathrm{HCC}$ patients were compiled; all of the patients were male, most of the HCC cases were caused by HBV infection, and only 2 cases exhibited an absence of hepatitis virus infection, including $\mathrm{HCV}$ and no liver cirrhosis. Only 2 cases with liver function were classified as Child-Pugh B, and most cases were Child-Pugh A. The average total diameter was $7.0 \mathrm{~cm}$, and the average largest tumor diameter was $6.2 \mathrm{~cm}$; most of these patients had a solitary tumor diameter ( 15 cases, $60 \%$ ), and 4 cases had multiple tumor targets (i.e., more than 3 targets). The AFP levels ranged from 0 to $400 \mathrm{ng} / \mathrm{ml}$ in 13 cases, from 400 to $800 \mathrm{ng} / \mathrm{ml}$ in 3 cases, and were more than $1210 \mathrm{ng} / \mathrm{ml}$ in 9 cases; most of the tumors in these patients ( 21 cases, $84 \%$ ) were stage III according to the TNM staging system (12 cases with IIIa, 9 cases with IIIb).

\section{Tumor recurrence and long-term survival}

The overall survival and tumor-free survival rates in these 25 cases are shown in Figure 1A and 1B. In the months following hepatic resection, 19 (76\%) of the 25 cases were diagnosed with HCC recurrence or metastasis; the most common site of HCC recurrence was the liver (14 cases), followed by liver recurrence and lung metastasis ( 2 cases), only lung metastasis (1 case), liver recurrence and bone metastasis (1 case), and liver recurrence and

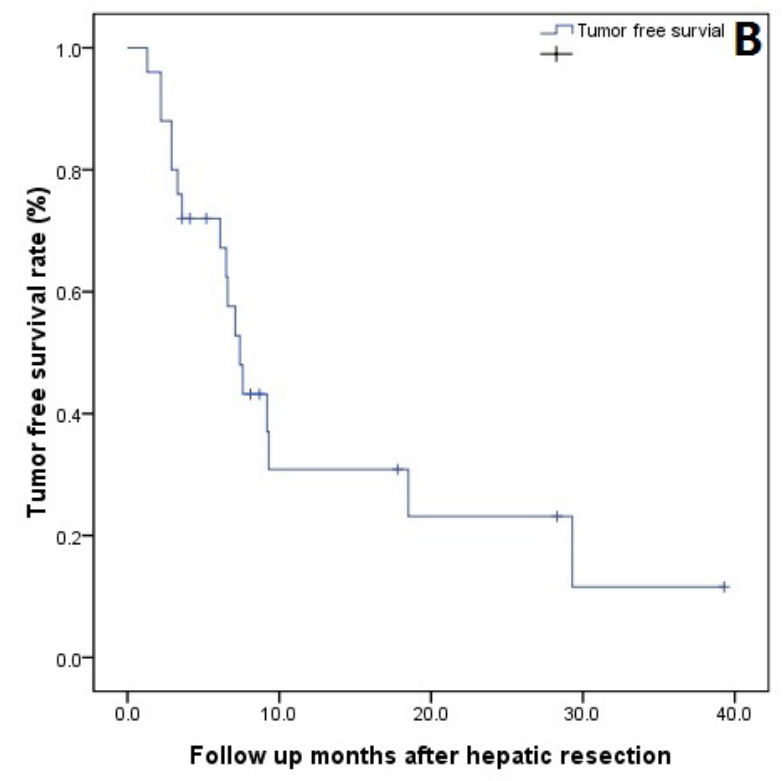

Figure 1: A-B: The overall. A. and tumor-free survival; B. following hepatic resection in 25 HCC patients. 
Table 1: Baseline demographic and tumor characteristics of the 25 patients.

\begin{tabular}{ll} 
Patient number & 25 \\
Age & $50.3 \pm 10.1$ \\
Gender $(\mathrm{M} / \mathrm{F})$ & $25 / 0$ \\
Height $(\mathrm{cm})$ & $170.4 \pm 4.3$ \\
Weight $(\mathrm{kg})$ & $69.5 \pm 8.6$ \\
BMI $\left(\mathrm{kg} / \mathrm{m}^{2}\right)$ & $24.8 \pm 4.2$ \\
Underlying liver disease (HBV/Negative) & $23 / 2$ \\
HBV-DNA (+/-) & $14 / 11$ \\
Child-Pugh score (A/B) & $23 / 2$ \\
Hemoglobin(g/L) & $142.3 \pm 20.9$ \\
Platelet $\left(\times 10^{9} / \mathrm{L}\right)$ & $143.2 \pm 62.9$ \\
Creatine & $81.4 \pm 10.2$ \\
Total bilirubin & $16.3 \pm 7.7$ \\
ALB (g/L) & $40.8 \pm 4.9$ \\
NLR(<4/ 4 ) & $21 / 4$ \\
Total tumor size(cm) & $7.0 \pm 2.6$ \\
Largest tumor size (cm) & $6.2 \pm 2.7$ \\
Tumor number (1/2/3/multiple) & $15 / 5 / 1 / 4$ \\
AFP level (-/+/++/+++) & $13 / 3 / 0 / 9$ \\
Risk factor (MI/STs/RMLT1/multiple) & $10 / 1 / 11 / 3$ \\
TNM stage (I/II/III) & $1 / 3 / 21$ \\
\hline
\end{tabular}

M: male; F: female; BMI: body mass index; HBV: hepatitis B virus; HCV: hepatitis C virus; AFP: alpha-fetoprotein; NLR: neutrophil/lymphocyte ratio; ALB: albumin; AFP level: -, 0-400 ng/ml; +, 400-800 ng/ml; ++, 800-1200 ng/ml; +++, $\geq 1210 \mathrm{ng} / \mathrm{ml}$; MI: microvascular invasion; STs: satellite targets; RMLT1: resection margins less than 1 cm; TNM: tumor node metastasis. 
Table 2: Univariate analyses showing factors that contribute to overall survival and tumor-free survival following LR.

\begin{tabular}{|c|c|c|c|}
\hline Variables & $\mathbf{N}$ & $\begin{array}{l}\text { Overall } \\
\text { survival }\end{array}$ & $\begin{array}{l}\text { Tumor-free } \\
\text { survival }\end{array}$ \\
\hline & & $P$-value & $P$-value \\
\hline Age $\leq 60($ yes/no $)$ & $20 / 5$ & 0.006 & 0.044 \\
\hline $\mathrm{BMI} \geq 28($ yes/no $)$ & $7 / 18$ & 0.634 & 0.671 \\
\hline Cause of liver diseases (HBV/no) & $23 / 2$ & 0.640 & 0.505 \\
\hline HBV-DNA (+/-) & $14 / 11$ & 0.696 & 0.524 \\
\hline Child-Pugh Score (A/B) & $23 / 2$ & 0.319 & 0.605 \\
\hline $\mathrm{Hb} \geq 120 \mathrm{~g} / \mathrm{L}$ (yes/no) & $21 / 4$ & 0.277 & 0.322 \\
\hline PLT $\geq 100 \times 10^{9} / \mathrm{L}($ yes $/$ no $)$ & $15 / 10$ & 0.976 & 0.975 \\
\hline Creatinine $\geq 90 \mu \mathrm{mol} / \mathrm{L}(\mathrm{yes} / \mathrm{no})$ & $2 / 23$ & 0.696 & 0.467 \\
\hline Total bilirubin $\geq 30 \mu \mathrm{mol} / \mathrm{L}$ (yes/no) & $2 / 23$ & 0.613 & 0.533 \\
\hline ALB level $\geq 40$ g/L (yes/no) & $17 / 8$ & 0.639 & 0.168 \\
\hline NLR $\geq 4$ (yes/no) & $4 / 21$ & 0.569 & 0.317 \\
\hline $\mathrm{AFP} \geq 800 \mathrm{ng} / \mathrm{ml}$ (yes/no) & $9 / 16$ & 0.686 & 0.417 \\
\hline Total tumor number (1/2-multiple) & $21 / 4$ & 0.622 & 0.407 \\
\hline Total tumordiameter $\geq 6 \mathrm{~cm}$ (yes/no) & $16 / 9$ & 0.756 & 0.777 \\
\hline Largest tumor size $\geq 6 \mathrm{~cm}($ yes $/ \mathrm{no})$ & $12 / 13$ & 0.239 & 0.243 \\
\hline Risk factors(MI/ST/RMLT1/multiple) & $10 / 1 / 11 / 3$ & 0.067 & 0.663 \\
\hline TNM stage (I/II/III) & $1 / 3 / 21$ & 0.602 & 0.585 \\
\hline VEGFR-2 (lower/higher) & $12 / 13$ & 0.395 & 0.956 \\
\hline VEGFR-3 (lower/higher) & $12 / 13$ & 0.539 & 0.671 \\
\hline PDGFR- $\beta$ (lower/higher) & $16 / 9$ & 0.370 & 0.172 \\
\hline c-Raf-1 (lower/higher) & $13 / 12$ & 0.004 & 0.020 \\
\hline
\end{tabular}

Abbreviations: M: male; F: female; BMI: body mass index; HBV: hepatitis B virus; NLR: neutrophil/ lymphocyte ratio; AFP: alpha-fetoprotein; PLT: platelet; Hb: hemoglobin; VEGFR: vascular endothelial growth factor receptor; PDGFR- $\beta$ : platelet-derived growth factor receptor; MI: microvascular invasion; ST: satellite targets; RMLT1: resection margins less than $1 \mathrm{~cm}$; TNM: tumor node metastasis. 
abdominal lymph node metastasis (1 case). To date, 17 cases have died, and all of the deaths in these patients were caused by $\mathrm{HCC}$ recurrence or metastasis. Additionally, the most common treatments for recurrence or metastasis were TACE (13 cases), TACE combined with RFA (2 cases), re-hepatic resection ( 2 cases), or TACE combined with LT (1 case).
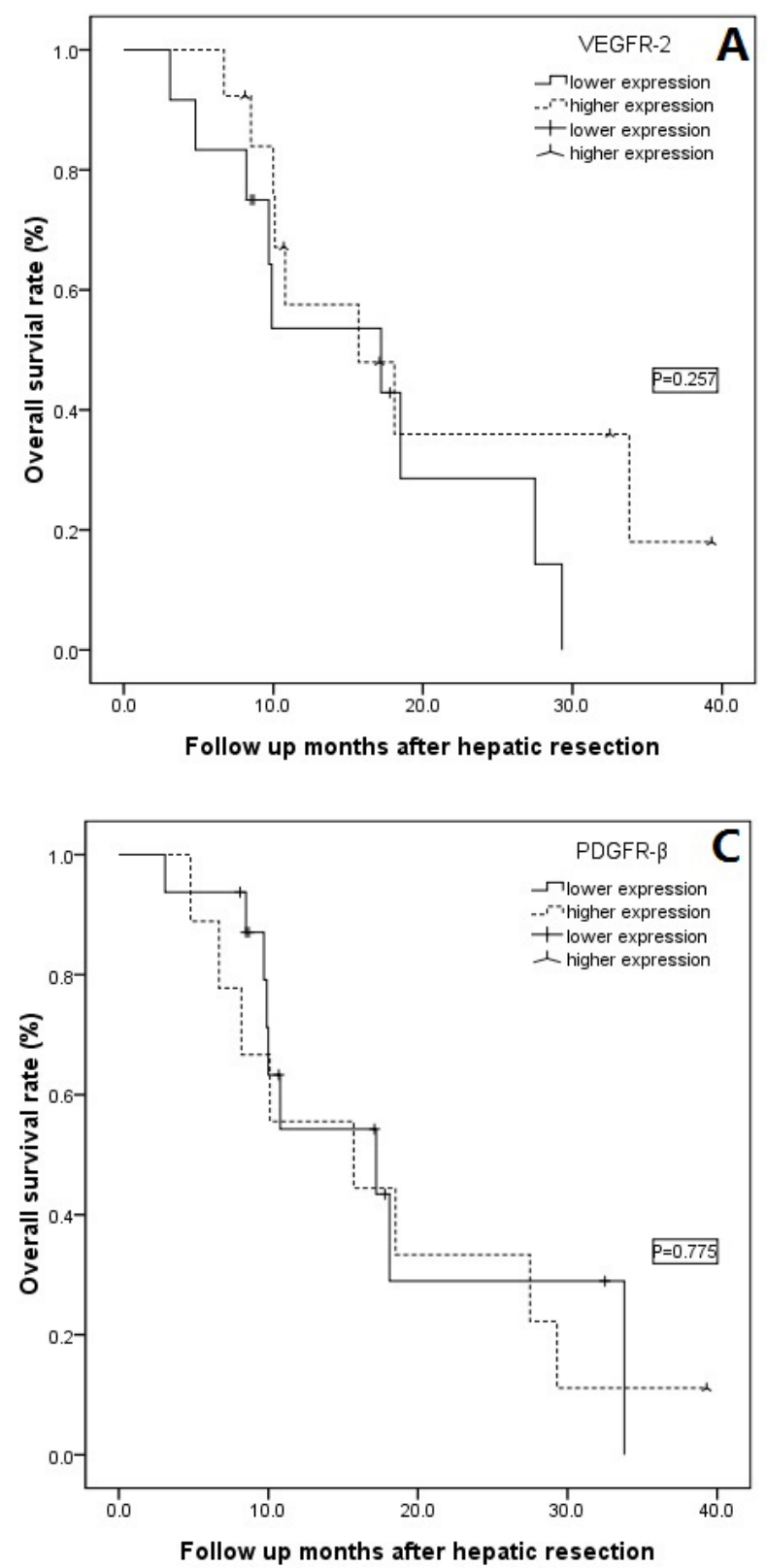

\section{Levels of tumor histological biomarkers}

Using a grading system based on the levels of tumor histological biomarkers, all 25 of the cases were divided into two groups: a low-expression (0-3) group or a high-expression (4-7) group using the four markers VEGFR-2/3, PDGFR- $\beta$ and c-Raf-1 (Figure 2). The overall survival rate showed no significant difference
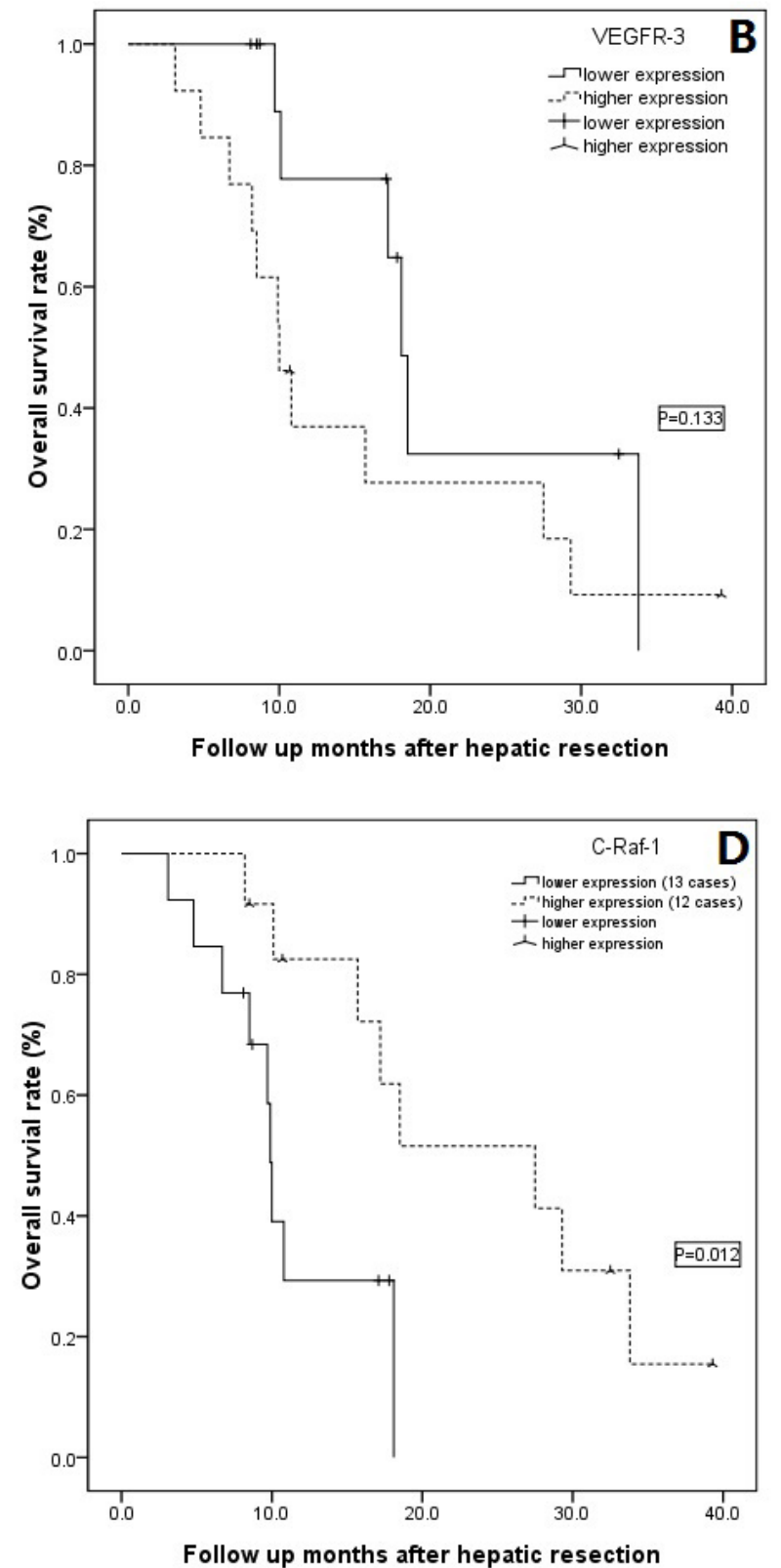

Figure 2: A-D: Subgroup overall survival analysis according to VEGFR-2/3, PDGFR- $\beta$ and c-Raf-1 expression. A. No difference was detected in the overall survival between the VEGFR-2 low- and high-expression groups $(P=0.257)$; B. No difference was detected in the overall survival between the VEGFR-3 low- and high-expression groups $(P=0.133)$; C. No difference was detected in the overall survival between the PDGFR- $\beta$ low- and high-expression groups $(P=0.775)$; D. The cases with high levels of c-Raf-1 expression showed significantly higher overall survival than the cases with lower levels of this biomarker $(P=0.012)$. 
between the low- and high-expression VEGFR-2/3 and PDGFR- $\beta$ subgroup analyses (Figure $2 \mathrm{~A}-2 \mathrm{C}$, all $P>$ $0.05)$; however, the high c-Raf-1 expression group showed significantly longer overall survival than the low c-Raf-1 expression group (Figure 2D, $P=0.012$ ). The advantage in long-term tumor-free survival in the high c-Raf-1 expression patients disappeared compared with the low c-Raf-1 expression patients (Figure 3, $P=0.061$ ).

\section{Univariate and multivariate analyses}

Additional survival analysis was performed (Table 2) that included 21 factors linked to survival: patient age, BMI, underlying liver disease, HBV-DNA, Child-Pugh score, Hb, PLT, creatine, TB, ALB, NLR, total tumor size, largest tumor size, tumor number, AFP level, risk factors, TNM stage, VEGFR-2, VEGFR-3, PDGFR- $\beta$, and c-Raf-1. Univariate analysis was performed using the data shown in Table 2, and patient age equal or younger than 60 years and higher levels of c-Raf-1 expression were prognostic factors that predicted both poor overall survival and tumor-free survival. Multivariate Cox regression analyses were performed for these two significant factors, and only higher expression of c-Raf-1 was a significant good factor for overall survival (HR: 1.842, 95\% CI: 1.211-2.542, $P=0.031$ ) and tumor-free survival (HR: $1.319,95 \%$ CI: $1.017-1.543, P=0.046)$ in HCC patients who underwent radical hepatic resection followed by sorafenib administration.

\section{DISCUSSION}

Hepatic resection has been used as the most common acceptable radical therapy for HCC; however, $\mathrm{HCC}$ recurrence remains the main problem following $\mathrm{HCC}$ resection. It is known that the presence of vascular invasion, satellite nodules, and resection margins less than $1 \mathrm{~cm}$ are significant predictive factors for tumor recurrence in HCC patients [20, 21]. Herein, all of the patients had at least one high-risk factor (microvascular invasion, satellite targets, or resection margins less than 1 $\mathrm{cm}$ ) for early recurrence, so sorafenib was recommended to all of these patients as a postoperative adjuvant therapy. Although some investigators have reported that preoperative therapies such as chemoembolization or chemotherapy do not show clinical benefit in terms of the prevention of relapse [22, 23], Llovet [24] advocated that research efforts should address the use of local or combination therapies in the adjuvant setting following resection. Angiogenesis plays an integral role in the progression of highly vascular malignancies such as HCC. Some key mediators of these pathways include the following: VEGFR, Raf, and PDGFR. These proteins have increasingly become important for the treatment of HCC because they are targeted by multikinase inhibitors [25].

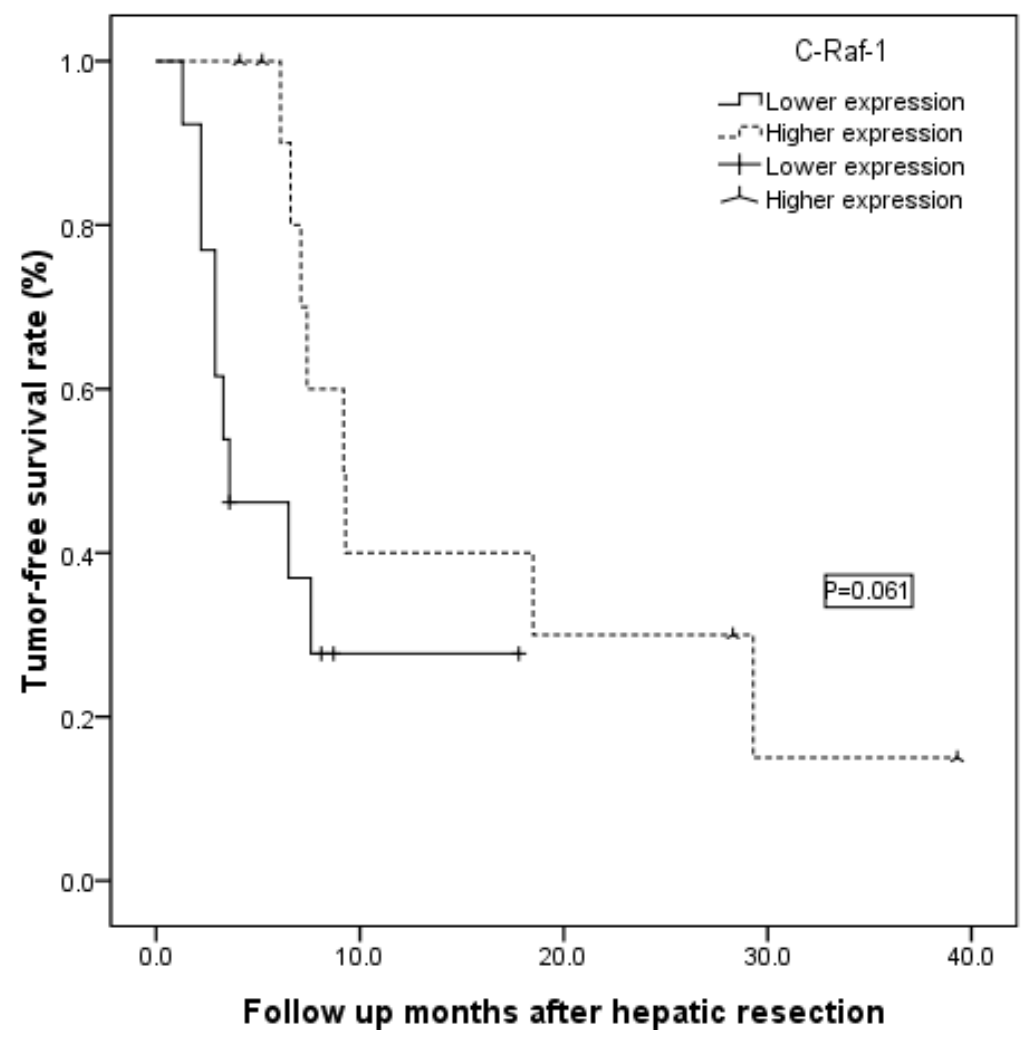

Figure 3: The patients with high levels of c-Raf-1 expression showed similar tumor-free survival rates compared with patients with low levels of c-Raf-1 expression $(P=0.061)$. 
Sorafenib is a multiple kinase inhibitor that specifically acts on VEGFR 2-3, PDGFR- $\beta$ and Raf kinases (Raf1) and prolongs survival time in patients with advanced HCC [12]. Sorafenib as an adjuvant therapy after curative resection has failed in a global, phase III, randomized, double-blind placebo-controlled clinical trial. However, the STORM trail showed that, although not all of the included patients benefit from adjuvant Sorafenib after radical resection, some patients did benefit from this combined therapy. Therefore, in the present study, we analyzed our 25 patients and found that the HCC patients with a higher level of c-Raf-1 expression might benefit from combined hepatic resection and adjuvant Sorafenib. This finding might indicate that the utility of Sorafenib is higher in cases that express high levels of c-Raf-1.

C-Raf-1 is thought to mainly have oncogenic potential and has been found to be highly expressed in certain human and animal malignancies, including HCC [26]. Although the overexpression of c-Raf-1 begins early in a preneoplastic lesion, it is predominantly expressed in basophilic tumors [26]. In the present study, all of the cases showed a high risk for HCC recurrence, even after undergoing radical hepatic resection. Accordingly, these patients were recommended to take Sorafenib as adjuvant therapy; 2 cases with a grade $3 \mathrm{AE}$ who could not tolerate this treatment were excluded from our study, but the remaining 25 cases were continued on Sorafenib therapy. When we compared the long-term outcomes, including overall survival and tumor-free survival, between subgroups, we found only that the cases with high levels of c-Raf-1 expression showed higher overall survival. Although cases with higher levels of c-Raf-1 expression showed increased tumor-free survival compared with cases with lower c-Raf-1 expression levels, this difference did not reach statistical significance. Meanwhile, the univariate and multivariate analyses also showed that lower levels of c-Raf-1 expression were a risk factor for lower overall and tumor-free survival. Our findings have some support from animal experiments. Wang et al. [27] reported that sorafenib inhibited tumor growth and prevented metastatic recurrence after resection in nude HCC mice. Feng et al. [28] examined the role of sorafenib in the prevention of HCC recurrence and found that sorafenib suppressed the development of postsurgical intrahepatic recurrence and abdominal metastasis, which led to prolonged postoperative survival of mice in this model [29].

The main limitations of this study were its limited sample size and retrospective nature. However, to overcome these limitations, and based on the result of this present study, we are now performing a large-sample, prospective study. Additionally, the median follow-up time in this report might not have long enough, so we would like to extend the follow-up time to at least 3 years for each case to observe the long-term outcomes.

In conclusion, patients with higher levels of c-Raf-1 expression on histological analysis might benefit from the administration of sorafenib after hepatic resection for cases with a high risk of tumor recurrence.

\section{MATERIALS AND METHODS}

We retrospectively collected HCC cases from Jan 2010 to Dec 2012; all of these inclusive cases accepted radical liver resection and then received Sorafenib as a postoperative adjuvant therapy. The ethical conduct of this study was approved by our departmental review board in agreement with the 1990 Declaration of Helsinki and its subsequent amendments. The diagnosis of HCC was made on the basis of a positive serum AFP level $(>400 \mathrm{ng} / \mathrm{ml})$ with positive imaging findings or at least two enhanced imaging techniques (ultrasound, CT or MRI) showing characteristic findings of arterial hypervascularization in all or part of the tumor and hypoattenuation in the portalvenous phase in high-risk patients $[16,17]$. The CT or MRI diagnosis of HCC was based on the presence of lesions with different echogenicity-hypoechoic, hyperechoic, isoechoic, or a mixed pattern-compared with the surrounding liver parenchyma. Livers were examined for tumor size and number, histologic differentiation, and the presence of microvascular and perineural invasion on histological examination. The inclusion criteria included the following: age from 18 to 70; resectable $\mathrm{HCC}$; Child-Pugh score of A or B; histological confirmation of HCC with a high risk of recurrence and with MI, STs or RMLT1; and treatment by liver resection and postoperative sorafenib. The exclusion criteria included the following: cardiovascular or cerebrovascular disease that was a surgical contraindication; coagulation disorders; bile duct-derived or mixed liver cancer; having undergone liver transplantation or other radical therapy; or only willing to undergo radical therapy with no postoperative sorafenib. Based on the inclusion and exclusion criteria, $25 \mathrm{HCC}$ cases were included in our present study.

We retrospectively collected data from these 25 cases and analyzed the risk factors that could contribute to tumor recurrence after liver resection and following sorafenib therapy, including baseline demographic characteristics, tumor characteristics, and tumor histological biomarkers-VEGFR-2/3, PDGFR- $\beta$ and c-Raf-1. The percentages of positive cells were scored into four categories according to staining: 0 for $0 \%, 1$ for $1-25 \%, 2$ for $26-50 \%, 3$ for $51-75 \%$, and 4 for $76-100 \%$. Additionally the staining intensities were also scored into four grades: $0,1,2$, and 3 . The sum of the percentages and intensity scores was used as the final staining score as described previously [18, 19]. Scores were defined as follows: $0-1$, negative; 2-3, low expression; 4-5, moderate expression; and 6-7, high expression. Cases were divided into two groups: a low-expression (0-3) group and a high-expression (4-7) group.

All of the surgical resections were conducted by 
laparotomy with a standard resection of the liver lobe or liver segment using the clamp method. At our center, surgeries are carried out by the chief physician or a deputy chief physician who has more than ten years of surgical experience. The resection uses the clamp method or an ultrasonic knife to implement the standard resection of a liver lobe or liver segment. The resection section was at least $1 \mathrm{~cm}$ from the tumor border. Intraoperative in vivo radiotherapy and chemotherapy were not applied, and no portal vein chemotherapy was provided. During surgery, we applied B-mode ultrasound at the same time for intra-operative tumor localization and examination after resection.

Sorafenib was administered to the 25 cases beginning two weeks after liver resection. The initial dosage was $400 \mathrm{mg}$ twice daily via mouth. Safety was assessed continuously, and adverse events and serious adverse events (AEs) were graded according to the National Cancer Institute Common Terminology Criteria for Adverse Events version 3.0. The low-dose level included $200 \mathrm{mg}$ twice daily, or withdrawal. Additionally, treatment for AEs, including observation for grade 1 and 2 and dose reduction or withdrawal for grades higher than 2 and then symptomatic treatment, were applied to these cases.

After discharge, follow-up was performed monthly in the 3 months after liver resection, every 2-3 months in the first year, and then every 3-5 months thereafter in the HCC cases. Patients also came to the clinic at our hospital for relevant monthly follow-up examinations, including abdominal ultra-sonography, AFP, liver function testing, and regular blood examinations. Additionally, routine ultra-sonography of the abdomen was performed to check for the recurrence of the tumor every 2-3 months. When the color ultra-sonography indicated a suspicious recurrence, we advised the patients to undergo enhanced CT or MRI inspection in combination with measurement of AFP level. If a recurrence was detected, we recommended the relevant treatment plan (e.g., re-resection, re-RFA, or interventional surgery) to the patients based on their individual situation, the characteristics of the tumor, and other relevant factors. The overall follow-up time was defined as the interval between the first radical therapy and either local tumor progression or the last follow-up.

We used SPSS (SPSS Inc., Chicago, IL, USA) software version 17.0 for the statistical analysis of the data, which were presented as the mean \pm standard deviation for normally distributed data. For univariate analysis, we used Student's $t$-test for continuous variables, and the chi-squared test or Fisher's exact test was used to compare categorical variables. The Kaplan-Meier method was used to estimate the overall and tumor-free survival rates. Univariate and multivariate analyses were carried out using the Cox regression method. A P-value $<0.05$ was considered a statistically significant difference.

\section{Abbreviations}

HCC: Hepatocellular carcinoma, LT: liver transplantation; TACE: transarterial chemoembolization, NLR: Neutrophil/lymphocyte ratio, MRI: magnetic resonance imaging; CT: computed tomography; HBV DNA: hepatitis $\mathrm{B}$ virus deoxyribonucleic acid; BMI: body mass index; HBV: hepatitis B virus; HCV: hepatitis $C$ virus; MELD: model for end-stage liver disease; AFP: alpha-fetoprotein; RFA: radiofrequency ablation; M: man; F: female; ALB: albumin; MI: microvascular invasion; STs: satellite targets; RMLT1: resection margins less than $1 \mathrm{~cm}$; TNM: tumor node metastasis; VEGFR: vascular endothelial growth factor receptor; PDGFR- $\beta$ : plateletderived growth factor receptor beta.

\section{ACKNOWLEDGMENTS}

This study was supported by grants from: The National was supported by grants from:The National Sciences and Technology Major Project of China (2012ZX10002-016) and (2012ZX10002-017)

\section{CONFLICTS OF INTERESTS}

No benefits in any form have been received or will be received from a commercial party related directly or indirectly to the subject of this article. The paper is not based on a previous communication to a society or meeting.

\section{GRANT SUPPORT}

No.

\section{Ethical approval}

Not needed.

\section{Author contributions}

Wang Wentao proposed the study. Lei Jianyong, Zhong Jinjing and Hao Jincheng performed the research and wrote the first draft. Lei Jianyong, Zhong Jinjing and Hao Jincheng collected and analyzed the data. All authors contributed to the design and interpretation of the study and to further drafts. Wang Wentao is the guarantor. The first authors of this manuscript are Lei Jianyong, Zhong Jinjing and Hao jincheng. These three contributed equally to this study and should be co-first authors. 


\section{REFERENCES}

1. Parkin DM, Bray F, Ferlay J and Pisani P. Global cancer statistics, 2002. CA Cancer J Clin. 2005; 55:74-108.

2. Bruix J, Gores GJ and Mazzaferro V. Hepatocellular carcinoma: clinical frontiers and perspectives. Gut. 2014; 63:844-855

3. Vitale A, Morales RR, Zanus G, Farinati F, Burra P, Angeli P, Frigo AC, Del Poggio P, Rapaccini G, Di Nolfo MA, Benvegnu L, Zoli M, Borzio F, Giannini EG, Caturelli E, Chiaramonte M, et al. Barcelona Clinic Liver Cancer staging and transplant survival benefit for patients with hepatocellular carcinoma: a multicentre, cohort study. The Lancet Oncology. 2011; 12:654-662.

4. Zhong JH, Xiang BD, Gong WF, Ke Y, Mo QG, Ma L, Liu $\mathrm{X}$ and Li LQ. Comparison of long-term survival of patients with BCLC stage B hepatocellular carcinoma after liver resection or transarterial chemoembolization. PloS one. 2013; 8:e68193.

5. Zhong JH, Ke Y, Gong WF, Xiang BD, Ma L, Ye XP, Peng T, Xie GS and Li LQ. Hepatic Resection Associated With Good Survival for Selected Patients With Intermediate and Advanced-Stage Hepatocellular Carcinoma. Annals of surgery. 2014; 260:329-40.

6. Kooby DA, Egnatashvili V, Graiser M, Delman KA, Kauh J, Wood WC and Staley Iii CA. Changing management and outcome of hepatocellular carcinoma: evaluation of 501 patients treated at a single comprehensive center. Journal of surgical oncology. 2008; 98:81-88.

7. Ren ZG, Lin ZY, Xia JL, Ye SL, Ma ZC, Ye QH, Qin LX, Wu ZQ, Fan J and Tang ZY. Postoperative adjuvant arterial chemoembolization improves survival of hepatocellular carcinoma patients with risk factors for residual tumor: a retrospective control study. World journal of gastroenterology. 2004; 10:2791-2794.

8. Li F, Guo Z, Zhang Y, Wang H, Zhang X, Si T, Yu H and Qi L. Postoperative adjuvant arterial chemoembolization improves the survival of hepatitis B virus-related hepatocellular carcinoma: a retrospective control study. Irish journal of medical science. 2015; 184:753-9.

9. Kashiwazaki M, Hama N, Takiuchi D, Noguchi K, Hata T, Asukai K, Hamano R, Yoshioka S, Miki H, Tsujie M and Yano K. Resection and postoperative multidisciplinary treatment for hepatocellular carcinoma with massive portal venous tumor thrombus-a single-center experience [Article in Japanese]. Gan to kagaku ryoho. Cancer \& chemotherapy. 2013; 40:1675-1677.

10. Waghray A, Balci B, El-Gazzaz G, Kim R, Pelley R, Narayanan Menon KV, Estfan B, Romero-Marrero C and Aucejo F. Safety and efficacy of sorafenib for the treatment of recurrent hepatocellular carcinoma after liver transplantation. Clinical transplantation. 2013; 27:555-561.

11. Zhong JH, Ma L and Li LQ. Postoperative therapy options for hepatocellular carcinoma. Scandinavian journal of gastroenterology. 2014; 49:649-661.

12. Llovet JM, Ricci S, Mazzaferro V, Hilgard P, Gane E, Blanc JF, de Oliveira AC, Santoro A, Raoul JL, Forner A, Schwartz M, Porta C, Zeuzem S, Bolondi L, Greten $\mathrm{TF}$, Galle PR, et al. Sorafenib in advanced hepatocellular carcinoma. The New England journal of medicine. 2008; 359:378-390.

13. Zhu AX, Sahani DV, Duda DG, di Tomaso E, Ancukiewicz M, Catalano OA, Sindhwani V, Blaszkowsky LS, Yoon SS, Lahdenranta J, Bhargava P, Meyerhardt J, Clark JW, Kwak EL, Hezel AF, Miksad R, et al. Efficacy, safety, and potential biomarkers of sunitinib monotherapy in advanced hepatocellular carcinoma: a phase II study. Journal of clinical oncology. 2009; 27:3027-3035.

14. Faivre S, Raymond E, Boucher E, Douillard J, Lim HY, Kim JS, Zappa M, Lanzalone S, Lin X, Deprimo S, Harmon C, Ruiz-Garcia A, Lechuga MJ and Cheng AL. Safety and efficacy of sunitinib in patients with advanced hepatocellular carcinoma: an open-label, multicentre, phase II study. The Lancet Oncology. 2009; 10:794-800.

15. Koeberle D, Montemurro M, Samaras P, Majno P, Simcock M, Limacher A, Lerch S, Kovacs K, Inauen R, Hess V, Saletti P, Borner M, Roth A and Bodoky G. Continuous Sunitinib treatment in patients with advanced hepatocellular carcinoma: a Swiss Group for Clinical Cancer Research (SAKK) and Swiss Association for the Study of the Liver (SASL) multicenter phase II trial (SAKK 77/06). The oncologist. 2010; 15:285-292.

16. Bruix J, Sherman M and Practice Guidelines Committee AAftSoLD. Management of hepatocellular carcinoma. Hepatology. 2005; 42:1208-1236.

17. Bruix $\mathrm{J}$ and Sherman M. Diagnosis of Small HCC. Gastroenterology. 2005; 129:1364.

18. Tang Q, Liu YF, Zhu XJ, Li YH, Zhu J, Zhang JP, Feng $\mathrm{ZQ}$ and Guan XH. Expression and prognostic significance of the alpha B-crystallin gene in human hepatocellular carcinoma. Human pathology. 2009; 40:300-305.

19. Huang J, Zhang X, Tang Q, Zhang F, Li Y, Feng Z and Zhu J. Prognostic significance and potential therapeutic target of VEGFR2 in hepatocellular carcinoma. Journal of clinical pathology. 2011; 64:343-348.

20. Portolani N, Coniglio A, Ghidoni S, Giovanelli M, Benetti A, Tiberio GA and Giulini SM. Early and late recurrence after liver resection for hepatocellular carcinoma: prognostic and therapeutic implications. Annals of surgery. 2006; 243:229-235.

21. Plessier A, Codes L, Consigny Y, Sommacale D, Dondero F, Cortes A, Degos F, Brillet PY, Vilgrain V, Paradis V, Belghiti J and Durand F. Underestimation of the influence of satellite nodules as a risk factor for post-transplantation recurrence in patients with small hepatocellular carcinoma. Liver transplantation. 2004; 10:S86-90. 
22. Wu CC, Ho YZ, Ho WL, Wu TC, Liu TJ and P'Eng F K. Preoperative transcatheter arterial chemoembolization for resectable large hepatocellular carcinoma: a reappraisal. The British journal of surgery. 1995; 82:122-126.

23. Tung-Ping Poon R, Fan ST and Wong J. Risk factors, prevention, and management of postoperative recurrence after resection of hepatocellular carcinoma. Annals of surgery. 2000; 232:10-24.

24. Llovet JM and Bruix J. Novel advancements in the management of hepatocellular carcinoma in 2008. Journal of hepatology. 2008; 48 Suppl 1:S20-37.

25. Patel SH, Kneuertz PJ, Delgado M, Kooby DA, Staley CA, 3rd, El-Rayes BF, Kauh JS, Sarmiento JM, Hanish S, Cohen C, Farris AB, 3rd and Maithel SK. Clinically relevant biomarkers to select patients for targeted inhibitor therapy after resection of hepatocellular carcinoma. Annals of surgical oncology. 2011; 18:3384-3390.

26. Das T, Patra F and Mukherjee B. Effect of antisense oligomer in controlling c-raf.1 overexpression during diethylnitrosamine-induced hepatocarcinogenesis in rat. Cancer chemotherapy and pharmacology. 2010; 65:309318.
27. Wang Z, Hu J, Qiu SJ, Huang XW, Dai Z, Tan CJ, Zhou $\mathrm{J}$ and Fan J. An investigation of the effect of sorafenib on tumour growth and recurrence after liver cancer resection in nude mice independent of phosphorylated extracellular signal-regulated kinase levels. Expert opinion on investigational drugs. 2011; 20:1039-1045.

28. Feng YX, Wang T, Deng YZ, Yang P, Li JJ, Guan DX, Yao F, Zhu YQ, Qin Y, Wang H, Li N, Wu MC, Wang HY, Wang XF, Cheng SQ and Xie D. Sorafenib suppresses postsurgical recurrence and metastasis of hepatocellular carcinoma in an orthotopic mouse model. Hepatology. 2011; 53:483-492.

29. Wang SN, Chuang SC and Lee KT. Efficacy of sorafenib as adjuvant therapy to prevent early recurrence of hepatocellular carcinoma after curative surgery: A pilot study. Hepatology research : the official journal of the Japan Society of Hepatology. 2014; 44:523-531. 